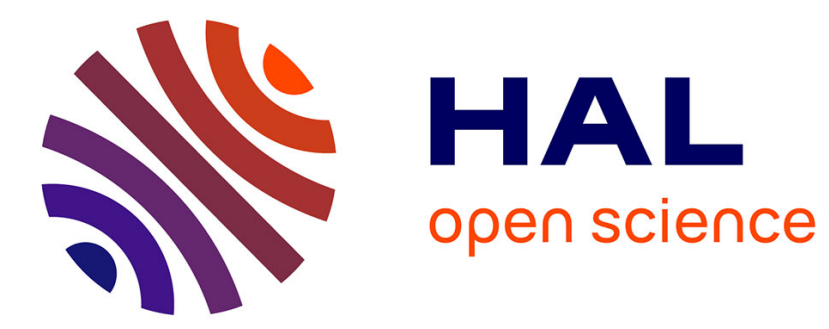

\title{
LECTURES ON PRINCIPAL BUNDLES
}

\author{
V. Balaji
}

\section{To cite this version:}

V. Balaji. LECTURES ON PRINCIPAL BUNDLES. 3rd cycle. Guanajuato (Mexique), 2006, pp.17. cel-00392133

\section{HAL Id: cel-00392133 \\ https://cel.hal.science/cel-00392133}

Submitted on 5 Jun 2009

HAL is a multi-disciplinary open access archive for the deposit and dissemination of scientific research documents, whether they are published or not. The documents may come from teaching and research institutions in France or abroad, or from public or private research centers.
L'archive ouverte pluridisciplinaire HAL, est destinée au dépôt et à la diffusion de documents scientifiques de niveau recherche, publiés ou non, émanant des établissements d'enseignement et de recherche français ou étrangers, des laboratoires publics ou privés. 


\title{
LECTURES ON PRINCIPAL BUNDLES
}

\author{
V. BALAJI
}

These are notes of five lectures given in Mexico in November 2006 at CIMAT, Guanajuato, in the "College on Vector Bundles" honour of Peter Newstead.

\section{INTRODUCTION}

The aim of these lectures is to give a brief introduction to principal bundles on algebraic curves towards the construction of the moduli spaces of semistable principal bundles. The first lecture develops the basic machinery on principal bundles, their automorphisms. At the end of the first chapter, we give a proof of theorem of Grothendieck on orthogonal bundles. The second chapter, after developing the notions of semistability and stability gives a modern proof of Grothendieck's theorem of classification of principal bundles on the projective line. The third chapter gives an outline of the construction of the moduli space of principal bundles on curves. The moduli space was constructed by A.Ramanthan in 1975. The method outlined here is from a new construction in [BS].

\section{Chapter 1}

Throughout these notes, unless otherwise stated, we have the following notations and assumptions:

(a) We work over an algebraically closed field $k$ of characteristic zero and without loss of generality we can take $k$ to be the field of complex numbers $\mathbb{C}$.

(b) $G$, will stand for a reductive algebraic group often the general linear group $G L(n)$ and $H$ a subgroup of $G$. Their representations are finite dimensional and rational.

(c) $X$ is a smooth projective curve almost always in these notes.

\subsection{Generalities on principal bundles.}

Definition 2.1.. A principal $G$ bundle $\pi: E \longrightarrow X$ with structure group $G$ (or a $G$ bundle for short) is a variety $E$ with a right $G$-action, the action being free, such that $\pi$ is $G$-equivariant, $X$ being given the trivial action. Further, the bundle $\pi$ is locally isotrivial, i.e, locally trivial in the étale topology.(in other words, for every $x \in X$, there exists a neighbourhood $U$ and an étale covering $U^{\prime} \longrightarrow U$ (i.e finite and unramified) such that, when $E$ is pulled back to $U^{\prime}$ it is "trivial" as $G$-bundle.

Remark 2.2.. In general we need to work with what is known as the fppf topology if the base is not smooth. 
- By a family of $H$ bundles on $X$ parametrised by $T$ we mean a principal $H$-bundle on $X \times T$, which we also denote by $\left\{E_{t}\right\}_{t \in T}$. We note that in general we may not have $T$ to be smooth and therefore we need the fppf local triviality.

- Recall the definitions of semisimple and reductive algebraic groups. Given an linear algebraic group $G$ define the radical $R(G)=(\bigcap B)^{0}$ the intersection running over all Borel subgroups, (which as we know are conjugate), or equivalently, $R(G)$ is the maximal, normal, connected, solvable subgroup of $G$. If $R(G)=(e)$ then $G$ is called semisimple, and if $R_{u}(G)$ the unipotent elements, called the unipotent radical of $G$ is trivial, then $G$ is called reductive.(In this case $R(G)$ will be a torus!) Equivalently, (by considering the derived subgroup) $G$ is semisimple (resp reductive) iff it has no connected abelian (resp unipotent abelian), normal subgroup other than $(e)$.

(a) Let $Y$ be any quasi projective $G$-variety and let $E$ be a $G$-principal bundle. For example $Y$ could be a $G$-module. Then we denote by $E(Y)$ the associated bundle with fibre type $Y$ which is the following object: $E(Y)=(E \times Y) / G$ for the twisted action of $G$ on $E \times Y$ given by $g .(e, y)=\left(e . g, g^{-1} . y\right)$.

(b) Any $G$-equivariant map $\phi: F_{1} \longrightarrow F_{2}$ will induce a morphism $E(\phi): E\left(F_{1}\right) \longrightarrow$ $E\left(F_{2}\right)$.

In particular, a section $s: X \longrightarrow E(F)$ is given by a morphism

$$
s^{\prime}: E \longrightarrow F
$$

such that, $s^{\prime}(e . g)=g^{-1} \cdot s^{\prime}(e)$ and $s(x)=\left(e, s^{\prime}(e)\right)$, where $e \in E$ is such that $\pi(e)=x$, where $\pi: E \longrightarrow X$.

Definition 2.3.. If $\rho: H \longrightarrow G$ is a homomorphism of groups the associated bundle $E(G)$, for the action of $H$ on $G$ by left multiplication through $\rho$, is naturally a $G$-bundle. We denote this $G$-bundle often by $\rho_{*}(E)$ and we say this bundle is obtained from $E$ by extension of structure group.

Definition 2.4. A pair $(E, \phi)$, where $E$ is a $H$-bundle and $\phi: E(G) \longrightarrow F$ is a $G$-bundle isomorphism, is said to give a reduction of structure group of the bundle $F$ to $H$. For convenience, we often omit $\phi$ and simply say $E$ is obtained from $F$ by reduction of structure group.

Two $H$-reductions of structure group $\left(E_{1}, \phi_{1}\right)$ and $\left(E_{2}, \phi_{2}\right)$ are equivalent or isomorphic if there is a $H$-bundle isomorphism $\psi: E_{1} \longrightarrow E_{2}$ such that the following diagram commutes:

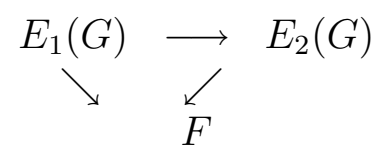

Remark 2.5.. A principal $G$-bundle $E$ on $X$ has an $H$-structure or equivalently a reduction of structure group to $H$ if we are given a section $\sigma: X \longrightarrow E(G / H)$, where $E(G / H) \simeq$ $E \times{ }^{G} G / H$. To see this, we note the identification of the spaces $E(G / H) \simeq E / H$. Then by pulling back the principal $H$-bundle $E \longrightarrow E / H$ by $\sigma$, we get an $H$-bundle $E_{H} \subset E$, giving the required $H$-reduction. In other words, there is a natural isomorphism $E_{H}(G) \simeq E$. Thus, we get a correspondence between sections of $E(G / H)$ and $H$-reductions of $E$. 
To see the other direction, let $E_{H}$ be a $H$-bundle and consider the natural inclusion $E_{H} \hookrightarrow E_{H}(G)=E$ given by $z \longrightarrow\left(z, 1_{G}\right)$, where of course $\left(z, 1_{G}\right)$ is identified with $\left(z h, h^{-1}\right)$ for $h \in H$. Going down by an action of $H$ we get a map $X \longrightarrow E_{H}(G) / H=E(G / H)$. This gives the required section of $E(G / H)$.

Remark 2.6. Note that a reduction of structure group of $E$ to $H \subset G$ can be realised by giving a $G$-map $s: E \longrightarrow G / H$ satisfying the propertys $(e . g)=g^{-1} s(e)$. In this sense, the reduction $E_{H}$ defined above can be seen to be the inverse image of the identity coset in $G / H$ by the map $s$.

Remark 2.7.. In the case of $G=G L(n)$, when we speak of a principal $G$-bundle we identify it often with the associated vector bundle by taking the associated vector bundle for the standard representation.

Remark 2.8.. A $G L(n)$ bundle is completely determined by the associated vector bundle $E(V)$, (where $V$ is the canonical $n$-dimensional space on which $G L(n)$ acts) as its bundle of frames. Let $\mathcal{V}$ be a vector bundle on $X$ with fibre the vector space $V$. Then consider the union

$$
\bigcup_{x \in X} \operatorname{Isom}\left(\mathcal{V}_{x}, V\right)
$$

where $\operatorname{Isom}\left(\mathcal{V}_{x}, V\right)$ are simply isomorphisms between the vector spaces $V$ and $\mathcal{V}_{x}$.

Note that there is a natural action of $G L(V)$ on the right which is easily seen to be free. This forms the total space of the principal $G L(V)$ bundle $E$ whose associated vector bundle $E(V) \simeq \mathcal{V}$

Similarly, a $P G L(n)$-bundle is equivalent to a projective bundle, i.e an isotrivial bundle with $\mathbf{P}^{\mathbf{n}}$ as fibre.

Proposition 2.9. . Let $E_{1}$ and $E_{2}$ be two $H$-bundles. Giving an isomorphism of the $H$ bundles $E_{i}$ is equivalent to giving a reduction of structure group of the principal $H \times H_{-}$ bundle $E_{1} \times_{X} E_{2}$ to the diagonal subgroup $\Delta_{H} \subset H \times H$.

Proof: A reduction of structure group to the diagonal $\Delta$ gives a $\Delta$-bundle $E_{\Delta}$. Now observe that the projection maps on $H \times H$ when restricted to the diagonal give isomorphisms of $\Delta \simeq H$. Seeing the bundle $E_{\Delta} \subset E_{1} \times_{X} E_{2}$ and using the two projections to $E_{1}$ and $E_{2}$, we get isomorphisms from $E_{1} \simeq E_{\Delta} \simeq E_{2}$. The converse is left as an exercise.

Definition 2.10.. Let $P$ be a $G$-bundle. Consider the canonical adjoint action of $G$ on itself, i.e $g \cdot g^{\prime}=g^{-1} g^{\prime} g$. Then we denote the associated bundle $P \times{ }^{G} G$ by $\operatorname{Ad}(\mathrm{P})$.

Observe that because of the presence of an identity section, the associated fibration $A d(P)$ is in fact a group scheme over $X$.

Proposition 2.11.. The sections $\Gamma(X, A d(P))$ are precisely the $G$-bundle automorphisms of $P$.

Proof: Let $\sigma: X \longrightarrow A d(P)$ be a section. We view the section $\sigma$ as remarked above as an equivariant map $\sigma: P \longrightarrow G$. Then by its definition, we have the following equivariance relation:

$$
\sigma(p . g)=g_{3}^{-1} \cdot \underset{3}{\sigma}(p)=g \sigma(p) g^{-1}
$$


Define the morphism:

$$
f_{\sigma}: P \longrightarrow P
$$

given by

$$
f_{\sigma}(p)=p \cdot \sigma(p)
$$

$\forall p \in P$. The equivariance property for $\sigma$ implies that $f_{\sigma}(p . g)=f_{\sigma}(p) \cdot g$ and hence its an $H$-morphism. Clearly it gives an automorphism of $P$.

For the converse, let $f: P \longrightarrow P$ be an $H$-bundles automorphism. Define $\sigma$ as follows:

$$
f(p)=p \cdot \sigma(p)
$$

Note that the $H$-equivariance property of $f$ implies the equivariance property of $\sigma$ which therefore defines a section of $\operatorname{Ad}(P)$.

Remark 2.12.. Let $E$ be a reduction of structure group of the principal $G$-bundle $P$ to the subgroup $H$. Then as we have remarked above, we can represent $E$ as a pair $(P, \phi)$ where $\phi: X \longrightarrow P(G / H)$ is a section of the associated fibration. Let $\sigma$ be an automorphism of the $G$-bundle $P$. Then, $\sigma$ also acts as an automorphism on the associated fibration $P(G / H)$. This gives an action of the group $A u t(P)$ on the set of all $H$-reductions of $P$.

Two $H$-reductions $E$ and $F$ of a principal $G$-bundle are equivalent (i.e give isomorphic $H$-bundles) iff there exists an automorphism $\sigma$ of $P$ which takes $E$ to $F$ in the above sense.

To illustrate this phenomenon I give below a theorem due to Grothendieck ([G]).

Theorem 2.13. Let $X$ be a smooth projective complex variety and let $H=O(n) \subset G=$ $G L(n)$ be the standard inclusion. Then the canonical map induced by extension of structure group

$$
\{\text { Isom classes of } H-\text { bundles }\} \longrightarrow\{\text { Isom classes of } G-\text { bundles }\}
$$

is injective. In other words, a $G$-bundle $P$ has, if any, a unique reduction of structure group to $H$ upto equivalence.

Proof: The proof of this theorem is quite beautiful and I will give it in full. Its also of importance to observe that the theorem is false for the inclusion $S O(n) \subset S L(n)$ !

Let $S$ be the space of symmetric $n \times n$-matrices which are non-singular. Then $G$ acts on $S$ as follows:

$$
\text { A.X }:=A X A^{t}
$$

The action is easily checked to be transitive and the isotropy subgroup at $I$ is the standard orthogonal group $H=O(n)$. i.e $S \simeq G / H$ as a $G$-space. The more important fact is that there is a canonical inclusion of $S$ in $G$. If $q: G \longrightarrow G / H$ is the canonical quotient map then identifying the quotient with $S$, the map $q$ is given by

$$
q(A)=A A^{t}
$$

and then the restriction of the map to $S \hookrightarrow G$ is given by the map $q_{S}(A)=A^{2}$ on the space of symmetric matrices. 


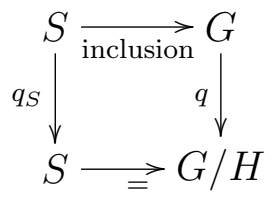

Let $P_{H}$ be a fixed reduction of structure group of $P$ to $H$ and let $E$ be any other reduction of $P$ which is therefore given by a section $\sigma: X \longrightarrow P(G / H)$. Since we already have a reduction, we can express the new reduction $\sigma$ as:

$$
\sigma: X \longrightarrow P_{H}(G / H)
$$

Consider the group scheme $A d(P)=P(G)$. Since $P$ has a $H$-reduction, we can view this group scheme as $P_{H}(G)$, where $H \hookrightarrow G$ acts on $G$ by conjugation i.e $h . g=h^{-1} g h$.

We also observe that the associated fibration $P_{H}(G / H)$ taken with the natural left action of $H$ on $G$ via its inclusion or by the conjugation action of $H$ on $G$ is identical. In other words, we can view the morphism associated to the canonical quotient map $G \longrightarrow G / H$ for the associated fibrations $P_{H}(G)$ and $P_{H}(G / H)$ as being induced by the conjugation action of $H$ on $G$ and $G / H$. Note that this is special to our situation since we have a $H$-reduction $P_{H}$ to start with.

We thus get the map:

$$
\phi: P_{H}(G) \longrightarrow P_{H}(G / H)
$$

Observe again that the space $S$ with its inclusion $S \hookrightarrow G$ is an $H$-morphism for the conjugation action of $H$. (Since $H=O(n), A^{t}=A^{-1}$ ).

Viewing the spaces in the diagram above as a diagram of $H$-spaces for the conjugation action we have the following diagram of associated spaces:

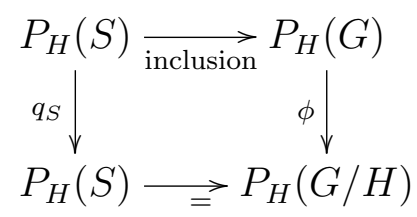

We now note that to prove that the reduction of $P$ given by $\sigma$ is equivalent to the one given by $P_{H}$, we need to give an automorphism which takes one to the other. An automorphism is giving a section of $P(G)$ or equivalently of $P_{H}(G)$. Its easy to check that, giving such an automorphism is giving a section $\gamma: X \longrightarrow P_{H}(G)$ such that the following diagram commutes:

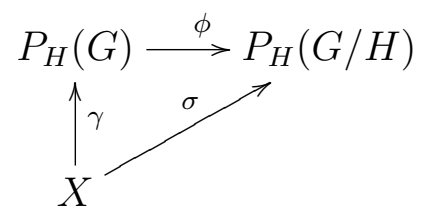

We now recall the following interpolation statement: 
Lemma 2.14.. The characteristic polynomial of a non-singular matrix $A$ can be used to get a square root of $A$.

Proof: By an interpolation exercise, we can construct a polynomial $h(t)$ such that $h(t)^{2}-t$ is divisible by $f(t)$, i.e $h^{2}(t)-t=f(t) g(t)$ for some polynomial $g(t)$. Since $f(A)=0$ by Cayley-Hamilton theorem, we get $h(A)^{2}=A$, i,e $h(A)$ provided a square root of $A$.

Now identify $P_{H}(G / H)$ with $P_{H}(S)$, which is a bundle of non-singular symmetric matrices. The section $\sigma$ gives a characteristic polynomial $f(t)$ with coefficients being holomorphic functions on $X$. Since $X$ is compact complex, these coefficients are therefore constant. Since these coefficients are constant, we can use the characteristic polynomial to get the square root of the section $\sigma$.

Take $h(t)$ as above. Then define

$$
\eta(x):=h(\sigma(x))
$$

This provided a section of $P_{H}(S)$ such that $\phi \circ \eta=\sigma$ since $\phi$ on $S$ is the squaring operation. The composition $\eta: X \longrightarrow P_{H}(S) \hookrightarrow P_{H}(G)$ gives the required $\gamma$.

Remark 2.15.. The reader should try and understand the proof in Grothendieck's paper. The main idea as suggested by Prof Ramanan, is to compare a pair of equivalent nondegenerate quadratic forms on a vector space. When carried out over a family, together with choosing a square root (over $X$ ) is essentially the classical proof. The proof given here applies the definitions developed here and also naturally generalises the problem.

Remark 2.16.. It fails for $S O(n) \subset S L(n)$. In fact, it fails for $n=2$. This can be seen as follows: $S O(2) \simeq \mathbf{G}_{m}$. Hence $S O(2)$ principal bundles cab be identifies with $\mathbf{G}_{m}$-bundles and hence with line bundles. Extension of structure group of a $S O(2)$-bundle to $S L(2)$ is equivalent to taking a line bundle $L$ to $L \oplus L^{*}$, which is an $S L(2)$-bundle. This has clearly two inequivalent reductions $L$ and $L^{*}$.

\section{Chapter 2}

Definition 3.1.. A vector bundle $V$ is said to be semistable (resp stable) if for every subbundle $W \subset V, \frac{\operatorname{deg}(W)}{r k(W)} \leq \frac{\operatorname{deg}(V)}{r k(V)}$.

Lemma 3.2. Let $V$ and $W$ be semistable vector bundles on $X$ of degree zero. Then $V \otimes W$ is semistable of degree zero. (Note that this theorem, as it stands is false in char p).

Proof. Any semistable bundle on $X$ of degree zero has a Jordan-Holder filtration such that its associated graded is a direct sum of stable bundles of degree zero. Note that the filtration is not unique but the associated graded is so. Hence the tensor product $V \otimes W$ gets a filtration such that its associated graded is a direct sum of tensor products of stable bundles of degree zero. We see easily that this reduces to proving the lemma when $V$ and $W$ are stable of degree zero. Then by the Narasimhan-Seshadri theorem, $V \otimes W$ is defined by a unitary representation of the fundamental group (namely the tensor product of the irreducible unitary representations which define $V$ and $W$ respectively), which implies that $V \otimes W$ is semistable (cf. Narasimhan-Seshadri, Annals of Math. 1965). 
Proposition 3.3.. Let $E$ be a principal $H$-bundle on $X$ with $H$ a semisimple algebraic group. Then the following are equivalent:

(a) There exists a faithful representation $H \hookrightarrow G L(V)$ such that the induced bundle $E(V)=E \times{ }^{H} V$ is semistable (of degree zero).

(b) For every representation $H \longrightarrow G L(W)$, the bundle $E(W)$ is semistable (of degree zero).

Proof.

(b) $\Longrightarrow(\mathrm{a})$ is obvious.

$(\mathrm{a}) \Longrightarrow(\mathrm{b})$ : Since $H$ is semisimple; the vector bundle $E(V)$ is semistable of degree 0 . Consider the natural tensor representation $T^{a, b}(V)=\otimes^{a} V \otimes \otimes^{b} V^{*}$. Then by Lemma 3.2, the bundle $E\left(T^{a, b}(V)\right)=\otimes^{a} E(V) \otimes \otimes^{b} E(V)^{*}$ is semistable of degree 0 .

It is well-known that any $H$-module $W$ is a sub-quotient of a suitable $T^{a, b}(V)$.

This can be seen as follows: Consider the action map $H \times W \longrightarrow W$. Taking the dual maps at the algebra level we have the map $W \longrightarrow k[H] \otimes W(0)$ where $W(0)$ is $W$ with the trivial $H$-action (namely h.w $=w \forall h \in H$ ). This follows by writing the action condition, where the action on the right is by translation on the algebra of functions. Hence, the $H$ module is a sub-module of $k[H]^{d i m W}$. In other words, it is enough to handle the $H$-module $k[H]$. For this embed $H \hookrightarrow G L(V)$ and embed $G L(V) \hookrightarrow \operatorname{End}(V) \times \operatorname{End}\left(V^{*}\right)$ (i.e inside matrices $(A, B)$, such that $\left.A \cdot B^{-1}=I\right)$. Then, by dualising and we see that

$$
\operatorname{Sym}(V) \otimes \operatorname{Sym}\left(V^{*}\right) \longrightarrow k[G L(V)] \longrightarrow k[H]
$$

the last two maps being surjections (H-maps). Now put $W$ in copies of $k[H]$ and we are done. Notice that if we are in char 0 then we can choose a splitting and embed $W$ in a finite direct sum of tensor representations.

Hence $E(W)$ is a sub-quotient of $E\left(T^{a, b}(V)\right)$ of degree zero. Therefore $E(W)$ is also semistable.

Definition 3.4.. An $H$ bundle $E$ is said to be semistable if it satisfies the equivalent conditions in Proposition 3.3.

\subsubsection{Tannakian definitions.}

Definition 3.5.. Another definition of a principal $G$-bundle is the Tannakian one: Let $X$ be a connected smooth projective variety over $\mathbf{C}$. Denote by $\operatorname{Vect}(X)$ the category of vector bundles over $X$. The category $\operatorname{Vect}(X)$ is equipped with an algebra structure defined by the tensor product operation

$$
\operatorname{Vect}(X) \times \operatorname{Vect}(X) \longrightarrow \operatorname{Vect}(X),
$$

which sends any pair $(E, F)$ to $E \otimes F$, and the direct sum operation $\bigoplus$, making it an additive tensor category in the sense of Definition 1.15 Deligne-Milne's article in Springer, LNM 900.

M.V.Nori gives an alternative description of principal $G$-bundles, which I briefly recall. For Nori however, $X$ is allowed to be a much more general space (a prescheme!). However 
we restrict ourselves to the situation where $X$ is a smooth variety, since the applications here will be in this generality.

Let $\operatorname{Rep}(G)$ denote the category of all finite dimensional complex left representations of the group $G$, or equivalently, left $G$-modules. By a $G$-module (or representation) we shall always mean a left $G$-module (or a left representation).

Given a principal $G$-bundle $P$ over $X$ and a left $G$-module $V$, the associated fiber bundle $P \times_{G} V$ has a natural structure of a vector bundle over $X$. Consider the functor

$$
F(P): \operatorname{Rep}(G) \longrightarrow \operatorname{Vect}(X)
$$

which sends any $V$ to the vector bundle $P \times^{G} V$ and sends any homomorphism between two $G$-modules to the naturally induced homomorphism between the two corresponding vector bundles. The functor $F(P)$ enjoys several natural abstract properties. For example, it is compatible with the algebra structures of $\operatorname{Rep}(G)$ and $\operatorname{Vect}(X)$ defined using direct sum and tensor product operations. Furthermore, $F(P)$ takes an exact sequence of $G$-modules to an exact sequence of vector bundles, it also takes the trivial $G$-module $\mathbf{C}$ to the trivial line bundle on $X$, and the dimension of $V$ also coincides with the rank of the vector bundle $F(P)(V)$.

Nori proves that the collection of principal $G$-bundles over $X$ are in bijective correspondence with the collection of functors from

$$
F: \operatorname{Rep}(G) \longrightarrow \operatorname{Vect}(X)
$$

satisfying the following properties: strict, exact, faithful,tensor functor such that $F_{x}$ is a fibre functor on the category $\operatorname{Rep}(G)$.

(a) Strict: a morphism of vector bundles is said to be strict if the cokernel is also locally free. Let $u: V \longrightarrow W$ be a $G$-module map. Then we want the induced morphism $F(u): F(V) \longrightarrow F(W)$ to be strict. In particular, $\operatorname{ker} F(u)$ and $\operatorname{Im} F(u)$ are also locally free.

(b) Exact: $\operatorname{ker} F(u)=F(\operatorname{ker}(u))$, $\operatorname{coker} F(u)=F(\operatorname{coker}(u))$.

(c) Faithful: $F(\operatorname{Hom}(V, W)) \hookrightarrow \operatorname{Hom}(F(V), F(W))$.

(d) Tensor functor: $F(V \otimes W)=V(V) \otimes F(W)$ and $F($ trivial $)=\mathcal{O}_{X}$.

(e) $F_{x}$ is a fibre functor, which says that using the pair $\left(\operatorname{Rep}(G), F_{x}\right)$, by the easy half of Grothendieck-Tannaka theorem one recovers back $G$ from the Tannakian category $\left(\operatorname{Rep}(G), F_{x}\right)$.

Given $F$, there is a $P$ unique upto a unique isomorphism such that $F \simeq F(P)$ (naturally equivalent!).

Remark 3.6.. More generally, this definition allows us to talk about torsors in any Tannaka category. For example, we could work with the category of semiharmonic Higgs bundles on a smooth projective variety, or the category of parabolic bundles with quasi-parabolic structure prescribed on a fixed divisor with simple normal crossings and the weights satisfying some natural conditions. Then a principal Higgs bundle or principle parabolic bundles could be defined as a functor satisfying Nori's axioms with values in the Higgs category or parabolic category (cf.[S]). This method can be used to construct moduli of these objects. 
Definition 3.7.. Fix a Borel subgroup $B$ and a maximal torus $T \subset B$. Let $P$ be a parabolic subgroup of the reductive group $G$. A character $\chi: P \longrightarrow \mathbf{C}^{*}$ is said to be dominant if it is trivial on the connected component of the centre of $G$ and such that with respect to positive roots $\alpha \in X^{*}(T)$, we have $\left(\alpha,\left.\chi\right|_{T}\right) \geq 0$. This notion does not depend on the choice of $B$. Here $\left(\right.$, ) denotes the natural W-invariant pairing on $X^{*}(T) \otimes \mathbf{Q}$.

Remark 3.8. Let $\chi$ be a character of $P$. Then, we consider the $P$-bundle $G \longrightarrow G / P$. The character $\chi$ then defines a line bundle $L_{\chi}$ on $G / P$. The property of dominance of $\chi$ is equivalent to the ampleness of the line bundle $L_{\chi}^{*}$.

Definition 3.9.. (A. Ramanathan)([R1], [R2]) $E$ is semistable if $\forall$ parabolic subgroup $P$ of $H, \forall$ reduction $\sigma_{P}: X \longrightarrow E(H / P)$ and $\forall$ dominant character $\chi$ of $P$, the bundle $\left.\sigma_{P}^{*}\left(L_{\chi}\right)\right)$ has degree $\leq 0$.

Theorem 3.10.. (A.Ramanathan) Let $H$ be semisimple. Then $E$ is semistable in the above sense if and only if it is so in the sense of Def 3.4.

Remark 3.11.. The proof of this theorem is quite non-trivial. I will indicate it at the end the course. We note that Ramanathan's definition makes sense for reductive groups as well.

Remark 3.12.. Recall the definition of stability of vector bundles. A vector bundle $V$ is said to be semistable (resp stable) if for every sub-bundle $W \subset V, \frac{\operatorname{deg}(W)}{r k(W)} \leq \frac{\operatorname{deg}(V)}{r k(V)}$. Comparing this definition with Ramanathan's definition given above it seems as if one is interested in asking Ramanathan's condition for maximal parabolic subgroups. This is indeed correct. It is not too hard, by expressing the dominant character of a given parabolic in terms of the characters of the maximal parabolic subgroups containing it, that one can always reduce to the case of maximal parabolic subgroups. Once on a maximal parabolic, then since $\operatorname{Pic}(G / P)=\mathbf{Z}$ it follows that there is only one inequality to be checked like the case of the vector bundles where we fail to see all the characters etc.

Remark 3.13.. We will, for the most part, be working with curves of $g \geq 2$. In $g=0$ we have the Grothendieck-Harder theorem (Harder for fields of char p) which says that all $G$-bundles have reduction of structure group to the maximal torus upto action by the Weyl group. This is true for any connected reductive group $G$.

Let $V$ be a vector bundle on $X$. Then recall that there is a unique filtration of $V$

$$
0=V_{0} \subset V_{1} \subset V_{2} \subset \ldots \subset V_{l-1} \subset V_{l}=V
$$

by subbundles and such that the filtration is characterised by the two conditions, namely, that all the quotients $V_{i} / V_{i-1}$ are semistable for $i \in[1, l]$ and further, the slopes $\operatorname{deg}\left(V_{i} / V_{i-1}\right) / \operatorname{rank}\left(V_{i} / V_{i-1}\right)$ are strictly decreasing as $i$ increases. This filtration was introduced by Harder and Narasimhan and is called the $H N$-filtration of $V$. This allows us to reduce, in some sense, all questions on vector bundles to semistable vector bundles. In the principal bundle setting we have the following:

Definition 3.14.. (Harder-Narasimhan Reduction) Let $E$ be a principal $G$ bundle on $X$ and $\left(P, \sigma_{P}\right)$ be a reduction of structure group of $E$ to a parabolic subgroup $P$ of $G$, then this reduction is called $\mathrm{H}-\mathrm{N}$ reduction if the following two conditions hold:

(a) If $L$ is the Levi factor of $P$ then the principal $L$ bundle $E_{P}(L)=E_{P} \times{ }^{P} L$ over $X$ is a semistable $L$ bundle. 
(b) For any dominant character $\chi$ of $P$ with respect to some Borel subgroup $B \subset P$ of $G$, the associated line bundle $L_{\chi}$ over $X$ has degree $>0$.

Remark 3.15.. For $G=G L(n, k)$ a reduction $E_{P}$ gives a filtration of the rank $n$ vector bundle associated to the standard representation. With a bit of work one can check that $E_{P}$ is canonical in the above sense iff the corresponding filtration of the associated vector bundle filtration coincides with its Harder Narasimhan filtration.

Theorem 3.16.. (Grothendieck)([G]) Let $E$ be a principal $G$-bundle on $\mathbf{P}^{\mathbf{1}}$, where $G$ is connected reductive. Then $E$ is has a reduction of structure group to the maximal torus of $G$ unique upto an action of the Weyl group.

Proof: The proof proceeds as follows. Case 1: $E$ is a semistable $G$-bundle.

Now we reduce further to the case when $G$ is semisimple, in which case Grothendieck's theorem would read:

$E$ semistable bundle with semisimple structure group, then $E$ is trivial.

For this, consider the quotient group $\bar{G}$ associated to $G$ by going modulo the identity component of the center. Then, since $E$ is semistable, it follows that $E(\bar{G})$ is also semistable. This needs a proof but is not difficult. (see A.Ramanathan. [R2])

Now $\bar{G}$ is semisimple we get by the semisimple version of Grothendieck that $E(\bar{G})$ is trivial. This implies that the structure group of $E$ reduces to the identity component of the center of $G$ proving the theorem.

To complete the proof we need to prove the result if the group $G$ is semisimple then the only semistable bundle will be the trivial one. Let $G \hookrightarrow S L(V)$ be a faithful representation. Then consider $E(V)$ the extension of structure group to $S L(V)$. Since $E(V)$ is semistable with trivial determinant, by Riemann-Roch, $E(V)$ has a section, which will be nowhere vanishing (because of the semistability and degree 0 property of $E(V)$ ). Since we are on $\mathbf{P}^{\mathbf{1}}$, we get a splitting of $E(V)$ with a trivial factor. Now an induction on rank of $E(V)$ proves that $E(V)$ is trivial. Since $E(V)$ comes with a reduction of structure group to $G$, we get a section of $E(S L(V)) \times{ }^{S L(V)} S L(V) / G$. Since $E(S L(V))$ is trivial, this section gives a map from $\mathbf{P}^{\mathbf{1}} \longrightarrow S L(V) / G$ which will be constant, since $S L(V) / G$ is an affine variety. This implies that $E$ is trivial, completing the proof.

Case 2: $E$ is not semistable. Then we reduce to the semistable case as follows:

Suppose that $E$ is unstable. Then by the existence of HN reduction, we have a parabolic subgroup $P \subset G$ and a reduction of structure group $E_{P}$ to $P$ such that the associated Levi bundle $E_{P}(L)$ is semistable. If we prove that $E_{P}(L)$ has a reduction of structure group to the maximal torus of $L$ then we observe that the parabolic subgroup is a semi-direct product of $L$ and the unipotent radical $U$. The point to note is that $U$ has a filtration by the additive group $\mathbf{G}_{\mathbf{a}}$ and hence on $\mathbf{P}^{\mathbf{1}}$, one can prove without much difficulty that $H^{1}\left(\mathbf{P}^{1}, U\right)=0$. This implies that the extended bundle $E_{P}(L)$ can in fact be realised as a bundle obtained by reduction of structure group to "a" Levi subgroup $L$ (by a choice of a splitting). In other words, if $E_{P}(L)$ has a reduction of structure group to its maximal torus, then so does $E$ to the maximal torus of $G$. 
Remark 3.17.. In the case of elliptic curves the same reduction statement is true for semistable bundles with vanishing Chern classes (true when $G$ is semisimple!) and the moduli is simply the $X^{k} / W$. In the case of vector bundles it is the symmetric product of the curve (Atiyah).

Definition 3.18.. A reduction of structure group of $E$ to a parabolic subgroup $P$ is called admissible if for any character $\chi$ on $P$ which is trivial on the center of $H$, the line bundle associated to the reduced $P$-bundle $E_{P}$ has degree zero.

Definition 3.19.. An $H$-bundle $E$ is said to be polystable if it has a reduction of structure group to a Levi $R$ of a parabolic $P$ such that the reduced $R$-bundle $E_{R}$ is stable and the extended $P$ bundle $E_{R}(P)$ is an admissible reduction of structure group for $E$. One can define the associated graded of a semistable $E$, denoted by $\operatorname{gr}(E)$ as follows: firstly one shows that there exists an admissible reduction of structure group to $P$ with the added property that $E_{P}(R)$ is stable as an $R$-bundle. (note that $R$ is only a reductive group and almost never semisimple!). Define $\operatorname{gr}(E) \simeq E_{P}(R)(G)$. This will be the unique closed orbit (in the GIT construction) in the orbit closure of $E$. In the case $G=G L(n), E$ the associated vector bundle. Then an example of an admissible reduction is writing $E$ as:

$$
0 \longrightarrow E_{1} \longrightarrow E \longrightarrow E_{2} \longrightarrow 0
$$

where $\operatorname{deg}\left(E_{i}\right)=0$. One knows by the theory of semistable bundles that in general $E$ has a filtration $E_{k} \subset \ldots \subset E_{1} \subset E$ of bundles such that the successive quotients $E_{i} / E_{i+1}$ are stable of degree 0 and then we define $\operatorname{gr}(E) \simeq \oplus E_{i} / E_{i+1}$.

(cf $\S 4$ (4.9) for other equivalent formulations).

\section{Chapter 3}

4.1. Construction of the moduli space. For the present purpose, we take $G=S L(n, \mathbb{C})$ and $H \subset G$ a semisimple subgroup. We will be interested in constructing a moduli space of $H$-bundles. Roughly, we wish to give the set $M_{H}$ of isomorphism classes of $H$-bundles the structure of an algebraic scheme in a natural way. Natural here could mean representing the functor $F_{H}$ or equivalently, given a family of $H$-bundles $F \longrightarrow X \times T$, parametrised by and algebraic scheme $T$, the natural set map

$$
t \longrightarrow\left(F_{t}\right)
$$

from

$$
T \longrightarrow M_{H}
$$

is a morphism. We note that there are bad bundles forcing us to refine our equivalence from isomorphism classes to $S$-equivalence. Indeed one can have a family of $H$-bundles $\left\{E_{t}\right\}$, on $\mathbf{A}^{1} \times X$, parametrised by the affine line, such that $\forall t \neq 0 \in \mathbf{A}^{1} E_{t} \simeq E$ and $E_{0} \simeq \operatorname{gr}(E)$. For this see the last part of construction where the points of the moduli are defined. The $S$-equivalence is in some sense identifying the general points on this family and the limit so as to make the resultant moduli a separated scheme.

We recall very briefly the Grothendieck Quot scheme used in the construction of the moduli space of vector bundles (cf.[N]). 
Let $\mathcal{F}$ be a coherent sheaf on $X$ and let $\mathcal{F}(m)$ be $\mathcal{F} \otimes \mathcal{O}_{X}(m)$ (following the usual notations). Choose an integer $m_{0}=m_{0}(n, d)(n=\mathrm{rk}, d=\operatorname{deg})$ such that for any $m \geq m_{0}$ and any semistable bundle $V$ of rank $n$ and $\operatorname{deg} d$ on $X$ and we have $h^{i}(V(m))=0$ and $V(m)$ is generated by its global sections.

Let $\chi=h^{0}(V(m))$ and consider the Quot scheme $Q$ consisting of coherent sheaves $\mathcal{F}$ on $X$ which are quotients of $\mathbb{C}^{\chi} \otimes_{\mathbb{C}} \mathcal{O}_{X}$ with a fixed Hilbert polynomial $P$. The group $\mathcal{G}=G L(\chi, \mathbb{C})$ canonically acts on $Q$ and hence on $X \times Q$ (trivial action on $X$ ) and lifts to an action on the universal sheaf $\mathcal{E}$ on $X \times Q$.

Let $R$ denote the $\mathcal{G}$-invariant open subset of $Q$ defined by

$$
R=\left\{\begin{array}{ll}
q \in Q \mid \begin{array}{l}
\mathcal{E}_{q}=\left.\mathcal{E}\right|_{X \times q} \text { is locally free s.t. the canonical map } \\
\mathbb{C}^{\chi} \longrightarrow H^{0}\left(\mathcal{E}_{q}\right) \text { is an isomorphism, } \operatorname{det} \mathcal{E}_{q} \simeq \mathcal{O}_{X} .
\end{array}
\end{array}\right\}
$$

We denote by $Q^{s s}$ the $\mathcal{G}$-invariant open subset of $R$ consisting of semistable bundles and let $\mathcal{E}$ continue to denote the restriction of $\mathcal{E}$ to $X \times Q^{s s}$.

Henceforth, 'by abuse of notation', we shall write $Q$ for $Q^{s s}$.

Definition 4.1.. Recall the definition of a good quotient. Let $H \times T \longrightarrow T$ be an action of $H$ on $T . p: T \longrightarrow Y=T / / H$ is called a good quotient if:

(a) $p$ is onto, affine and $H$-invariant.

(b) $p_{*}\left(\mathcal{O}_{T}^{H}\right)=\mathcal{O}_{Y}$ where $\mathcal{O}_{T}^{H}$ is the sheaf of $H$-invariant sections ...

(c) Closed $H$-subsets of $T$ map to closed subs of $Y$ and disjoint closed sets go to disjoint closed sets by $p$.

If all the orbits are closed then $p$ is called a geometric quotient. If further the action map $(H \times T) \longrightarrow(T \times T)$ is a closed embedding, in which case we say the action is free (in the strong sense!), then $p$ is an $H$-torsor or principal bundle. There can be situations where the action is set theoretically free and a geometric quotient when $p$ is not a principal bundle (cf Mumford's GIT). However, in char 0, by Luna one can always have an open subset where the action is strongly free and hence a principal bundle. (cf Newstead $[\mathrm{N}]$ )

4.2. The construction of the moduli space for principal bundles. Fix a base point $x \in X$ (cf. Remark 2.3). Let $q^{\prime \prime}:(\mathrm{Sch}) \longrightarrow$ (Sets) be the following functor:

$$
q^{\prime \prime}(T)=\left\{\left(V_{t}, s_{t}\right) \mid \begin{array}{l}
\left\{V_{t}\right\} \text { is a family of semistable principal } G \text {-bundles } \\
\text { parametrised by } T \text { and } s_{t} \in \Gamma\left(X, V(G / H)_{t}\right) \forall t \in T
\end{array}\right\} .
$$

i.e. $q^{\prime \prime}(T)$ consists pairs of rank $n$ vector bundles (or equivalently principal $G$ bundles) together with a reduction of structure group to $H$.

By appealing to the general theory of Hilbert schemes, one can show without much difficulty (cf. [R1, Lemma 3.8.1]) that $q^{\prime \prime}$ is representable by a $Q$-scheme, which we denote by $Q^{\prime \prime}$.

The universal sheaf $\mathcal{E}$ on $X \times Q$ is in fact a vector bundle. Denoting by the same $\mathcal{E}$ the associated principal $G$-bundle, set $Q^{\prime}=(\mathcal{E} / H)_{x}$. Then in our notation $Q^{\prime}=\mathcal{E}(G / H)_{x}$ i.e. we take the bundle over $X \times Q$ associated to $\mathcal{E}$ with fibre $G / H$ and take its restriction to 
$x \times Q \approx Q$. Let $f: Q^{\prime} \longrightarrow Q$ be the natural map. Then, since $H$ is reductive, $f$ is an affine morphism.

Observe that $Q^{\prime}$ parametrises semistable vector bundles together with initial values at $x$ of possible reductions to $H$.

Define the "evaluation map" of $Q$-schemes as follows:

$$
\begin{gathered}
\phi_{x}: Q^{\prime \prime} \longrightarrow Q^{\prime} \\
(V, s) \longmapsto(V, s(x)) .
\end{gathered}
$$

We then have the following two technical lemmas. (cf $[\mathrm{BS}]$ ) for a proof of the first one.

Lemma 4.2.. The evaluation map $\phi_{x}: Q^{\prime \prime} \longrightarrow Q^{\prime}$ is proper.

Lemma 4.3.. The evaluation map $\phi_{x}$ is injective.

Proof. Since $H$ is semisimple, it follows that $G / H$ is affine and by a classical result of Chevalley, we have a $G$-embedding $G / H \hookrightarrow W$ in a finite dimensional $G$-module $W$. Let $(E, s)$ and $\left(E^{\prime}, s^{\prime}\right) \in Q^{\prime \prime}$ such that $\phi_{x}(E, s)=\phi_{x}\left(E^{\prime}, s^{\prime}\right)$ in $Q^{\prime}$. i.e. $(E, s(x))=\left(E^{\prime}, s^{\prime}(x)\right)$. So we may assume that $E \simeq E^{\prime}$ and that $s$ and $s^{\prime}$ are two different sections of $E(G / H)$ with $s(x)=s^{\prime}(x)$.

Using $G / H \hookrightarrow W$, we may consider $s$ and $s^{\prime}$ as sections in $\Gamma(X, E(W))$. Observe that by definition $E$ being semistable of degree 0 , so is $E(W)$.

Recall the following fact:

If $E$ and $F$ are semistable vector bundles with $\mu(E)=\mu(F)$, then the evaluation map

$$
\phi_{x}: \operatorname{Hom}(E, F) \longrightarrow \operatorname{Hom}\left(E_{x}, F_{x}\right)
$$

is injective.

In our situation, $s$ and $s^{\prime} \in \operatorname{Hom}\left(\mathcal{O}_{X}, E(W)\right)$ and hence by $(*)$ since $\phi_{x}(s)=\phi_{x}\left(s^{\prime}\right)$, we get $s=s^{\prime}$, proving injectivity.

Remark 4.4.. It is immediate that the $\mathcal{G}$-action on $Q$ lifts to an action on $Q^{\prime \prime}$.

Recall the commutative diagram

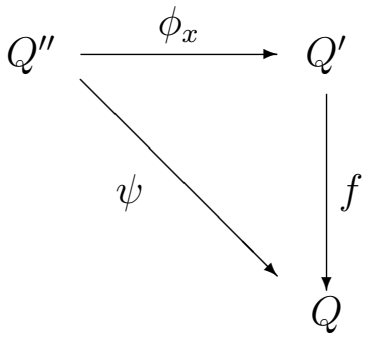

By Lemma 4.2, and Lemma $4.3 \phi_{x}$ is a proper injection and hence affine. One knows that $f$ is affine (with fibres $G / H)$. Hence $\psi$ is a $\mathcal{G}$ equivariant affine morphism.

Remark 4.5.. Let $(E, s)$ and $\left(E^{\prime}, s^{\prime}\right)$ be in the same $\mathcal{G}$-orbit of $Q^{\prime \prime}$. Then we have $E \simeq E^{\prime}$. Identifying $E^{\prime}$ with $E$, we see that $s$ and $s^{\prime}$ lie in the same orbit of Aut ${ }_{G} E$ on $\Gamma(X, E(G / H))$. Then using Remark 2.12 we see that the reductions $s$ and $s^{\prime}$ give isomorphic $H$-bundles. 
Conversely, if $(E, s)$ and $\left(E^{\prime}, s^{\prime}\right)$ such that $E \simeq E^{\prime}$ and the reductions $s, s^{\prime}$ give isomorphic $H$-bundles, using again Remark 2.12 we see that $(E, s)$ and $\left(E^{\prime}, s^{\prime}\right)$ lie in the same $\mathcal{G}$-orbit.

Consider the $\mathcal{G}$-action on $Q^{\prime \prime}$ with the linearisation induced by the affine $\mathcal{G}$-morphism $Q^{\prime \prime} \longrightarrow Q$. It is seen without much difficulty that, since a good quotient of $Q$ by $\mathcal{G}$ exists and since $Q^{\prime \prime} \longrightarrow Q$ is an affine $\mathcal{G}$-equivariant map, a good quotient $Q^{\prime \prime} / \mathcal{G}$ exists (cf. [N] Lemma 4.1).

Moreover by the universal property of categorical quotients, the canonical morphism

$$
\bar{\psi}: Q^{\prime \prime} / / \mathcal{G} \longrightarrow Q / / \mathcal{G}
$$

is also affine.

Theorem 4.6.. Let $M_{X}(H)$ denote the scheme $Q^{\prime \prime} / / \mathcal{G}$. Then this scheme is the coarse moduli scheme of semistable $H$-bundles. Further $M_{X}(H)$ is projective and if $H \hookrightarrow G L(V)$ is a faithful representation, the canonical morphism $\bar{\psi}: M_{X}(H) \longrightarrow M_{X}(G L(V))$ is finite.

Proof. We need only check the last statement. Theorem 4.10, which is termed the semistable reducton theorem) then implies that the moduli space $M_{X}(H)$ is projective, and therefore $\bar{\psi}$ is proper. By the remarks above $\bar{\psi}$ is also affine, therefore it follows that $\bar{\psi}$ is finite.

Remark 4.7. We have supposed that $H$ is semisimple; however, it is not difficult to treat the more general case when $H$ is reductive. Let $H$ be then reductive and $\bar{H}=H$ mod centre, its adjoint group. Let $P$ be a principal $H$-bundle and $\bar{P}$ the $\bar{H}$-bundle, obtained by extension of structure groups. We define $P$ to be semistable if $\bar{P}$ is semistable.

Recall that over a curve the topological type of the bundle is completely determined by $\pi_{1}(G)$. For example, consider the exact sequence (assume the $G$ is semisimple)

$$
1 \longrightarrow \pi_{1}(G) \longrightarrow G^{s c} \longrightarrow G \longrightarrow 1
$$

Then one observes that for the simply connected group, principal bundles are of one topological type (namely trivial!). Then follow the cohomology map

$$
H^{1}(X, \mathbf{G}) \longrightarrow H^{2}\left(X, \pi_{1}(G)\right) \simeq \pi_{1}(G)
$$

where, $\mathbf{G}$ is the sheaf of continuous functions with values in $G$. If we fix a topological isomorphism class $c$ for principal $H$-bundles, this fixes a topological isomorphism $\bar{c}$ for principal $\bar{H}$-bundles. Then the moduli space $M_{X}(H)_{c}$ is "essentially" $M_{X}(\bar{H})_{\bar{c}} \times$ (product of Jacobians). This can be made rigorous and leads to the construction of $M_{X}(H)_{c}$.

4.3. Points of the moduli. In this subsection we will briefly describe the $k$-valued points of the moduli space $M_{X}(H)$. The general functorial description of $M_{X}(H)$ as a coarse moduli scheme follows by the usual process.

Proposition 4.8.. The "points" of $M_{X}(H)$ are given by isomorphism classes of polystable principal $H$-bundles.

We firstly remark that since the quotient $q: Q^{\prime \prime} \longrightarrow M_{X}(H)$ obtained above is a good quotient, it follows that each fibre $q^{-1}(E)$ for $E \in M_{X}(H)$ has a unique closed $\mathcal{G}$-orbit. Let us denote an orbit $\mathcal{G} \cdot E$ by $O(E)$. The proposition will follow from the following: 
Lemma 4.9.. If $O(E)$ is closed then $E$ is polystable.

Proof. (Idea of proof) Recall the definition of a polystable bundle Def 3.19 and the definition of admissible reductions Def 3.18. If $E$ has no admissible reduction of structure group to a parabolic subgroup then it is polystable and there is nothing to prove.

Suppose then that $E$ has an admissible reduction $E_{P}$, to $P \subset H$. Recall by the general theory of parabolic subgroups that there exists a 1-PS $\xi: \mathbf{G}_{m} \longrightarrow H$ such that $P=P(\xi)$. Let $L(\xi)$ and $U(\xi)$ be its canonical Levi subgroup and unipotent subgroup respectively. The Levi subgroup will be the centraliser of this 1-PS $\xi$ and one knows $P(\xi)=L(\xi) \cdot U(\xi)=U(\xi) \cdot L(\xi)$. In particular, if $h \in P$ then $\lim \xi(t) \cdot h \cdot \xi(t)^{-1}$ exists. From these considerations one can show that there is a morphism

$$
f: P(\xi) \times \mathbf{A}^{1} \longrightarrow P(\xi)
$$

such that $f(h, 0)=m \cdot u$, where $h \in P$ and $h=m \cdot u, m \in L$ and $u \in U$. (see Lemma 3.5.12 A.Ramanathan)

Consider the $P$-bundle $E_{P}$. Then, using the natural projection $P \longrightarrow L$ where $L=L(\xi)$, we get an $L$-bundle $E_{P}(L)$. Again, using the inclusion $L \hookrightarrow P \hookrightarrow H$, we get a new $H$-bundle $E_{P}(L)(H)$. Let us denote this $H$-bundle by $E_{P}(L, H)$. It follows from the definition of admissible reductions and polystability that $E_{P}(L, H)$ is polystable.

Further, from the family of maps $f$ defined above, and composing with the inclusion $P(\xi) \hookrightarrow H$ we obtain a family of $H$-bundles $E_{P}\left(f_{t}\right)$ for $t \neq 0$ and all these bundle are isomorphic to the given bundle E. Following (Ramanathan Prop.3.5 pp 313), one can prove that the bundle $E_{P}(L, H)$ is the limit of $E_{P}\left(f_{t}\right)$. It follows that $E_{P}(L, H)$ is in the $\mathcal{G}_{\text {- }}$ orbit $O(E)$ because $O(E)$ is closed. This implies that $E \simeq E_{P}(L, H)$, implying that $E$ is polystable. Q.E.D.

To complete the projectivity of the moduli space, we need the following result. (cf [BS] and $[\mathrm{F}]$ for proofs).

Theorem 4.10. Let $P_{K}$ be a family of semistable principal $H$-bundles on $X \times$ Spec $K$, or equivalently, if $H_{K}$ denotes the group scheme $H \times$ Spec $K$, a semi-stable $H_{K}$-bundle $P_{K}$ on $X_{K}$. Then there exists a finite extension $L / K$, with the integral closure $B$ of $A$ in $L$, such that, $P_{K}$, after base change to $\operatorname{Spec} B$, extends to a semistable $H_{B}$-bundle $P_{B}$ on $X_{B}$.

Remark 4.11.. We outline in brief the classical Langton for vector bundles (cf [L]): We are given $V_{K}$ a semistable family on $X_{K}$. Firstly, there exists an extension of $V_{K}$ to a $V_{A}$ which we may choose as reflexive since we are on a family of curves. (the choice is quite non-canonical as can be seen from Prop 6 of Langton's paper). If the special fibre $V_{k}$ is not semistable., then let $F_{i}\left(V_{k}\right)$ be the first non-trivial term in the HN-filtration. We may assume that the extension $V_{A}$ has been chosen that $\mu\left(F_{i}\right)$ is minimal and also among these with the least $r k\left(F_{i}\right)$. We get a new model $V_{A}^{\prime}=\operatorname{ker}\left(V_{A} \longrightarrow V_{k} / F_{i}\left(V_{k}\right)\right)$ and an extension

$$
0 \longrightarrow V_{k} / F_{i}\left(V_{k}\right) \longrightarrow V_{k}^{\prime} \longrightarrow F_{i}\left(V_{k}\right) \longrightarrow 0
$$

From this, after some work, one shows that $F_{i}\left(V_{k}^{\prime}\right)$ injects into $F_{i}\left(V_{k}\right)$. Therefore by minimality, $F_{i}\left(V_{k}^{\prime}\right)=F_{i}\left(V_{k}\right)$. Continuing, one gets a decreasing family of models for $V_{K}$. Taking their intersection (making sense of it!) one gets a sub $W_{A} \subset V_{A}$ such that $W_{k} \simeq F_{i}\left(V_{k}\right)$. 
Thus, $\mu\left(W_{K}\right)=\mu\left(F_{i}\right)$, which is greater than $\mu\left(V_{K}\right)$, since $V_{k}$ is not semistable. This implies that $V_{K}$ itself is not semistable, a contradiction!

Remark 4.12.. The first crucial difficulty in proving our theorem is even at the very first step. Namely, there is no choice of extension of the family $E_{K}$ asa $H_{A}$ family or even as a torsor with a modified group scheme. If one chooses a representation then the associated vector bundle family via Langton's theorem has no canonical extension (be it the limit or the family!). Indeed, we cannot simply use Langton's theorem as we require the limiting bundle to be polystable, which can be ensured only by the GIT construction of the moduli space of vector bundles!

Remark 4.13.. Let $H \subset G$, where $G$ is a linear group. In the notation of $\S 2$ let $F_{H}$ and $F_{G}$ stand for the functors associated to families of semistable bundles of degree zero. (cf Proposition ??). The inclusion of $H$ in $G$ induces a morphism of functors $F_{H} \longrightarrow F_{G}$. We remark that the semistable reduction theorem for principal $H$-bundles need not imply that the induced morphism $F_{H} \longrightarrow F_{G}$ is a proper morphism of functors. Indeed, this does not seem to be the case. However, it does imply that the associated morphism at the level of moduli spaces is indeed proper (cf Theorem 4.6).

Remark 4.14.. We need to go to a ramified cover to extend our family unlike the vector bundle case. This can be seen in the following example: Let $\left(V_{A}, q_{A}\right)$ be a family of trivial vector bundles equipped with a non-degenerate quadratic form thus making it a family of orthogonal bundles. Consider the family $\left(V_{A} \oplus V_{A}, q_{A} \oplus t . q_{A}\right)$ where $t$ is the uniformising parameter. This is generically a family of orthogonal bundles the limit being the trivial bundle with degenerate quadratic form. The vector bundle family has trivially extended but the quadratic form fails to extend as a non-degenerate form. By going to the quadratic extension (totally ramified) $K(\sqrt{t})$ and letting $B$ be the integral closure of $A$ in this extension, we see that by modifying the quadratic form in its equivalence, namely $A . X . A^{t}$ using the matrix $A=\left(a_{i j}\right)$ where

$$
A=\left(\begin{array}{cc}
1 & 0 \\
0 & \sqrt{(t})^{-1}
\end{array}\right)
$$

\section{REFERENCES}

[BS] V.Balaji and C.S.Seshadri: Semistable Principal Bundles-I (characteristic zero) Journal of Algebra, 258 (2002), pp 321-347.

[DM] P.Deligne and D.Mumford: The irreducibility of the space of curves of a given genus, Publ I.H.E.S. 36 (1969) pp 75-109.

[F] G.Faltings: Stable G-bundles and projective connections, J. Alg. Geom., 2 (1993), 507-568.

[G] A.Grothendieck: Sur la classification des fibrés holomorphes sur la sphère de Riemann, Amer.J.Math. 79 (1956), 121-138.

[L] S.Langton: Valuative criterion for families of vector bundles on algebraic varieties, Annals of Mathematics (2) 101 (1975) pp 88-110.

[N] P.Newstead:Introduction to moduli problems an orbit spaces, Tata Inst. of Fundamental Research, Vol 51, Springer Verlag, 1978.

[R1] A. Ramanathan: Stable principal bundles on a compact Riemann surface - Construction of moduli space (Thesis), Bombay University 1976, Proc.Ind.Acad.Sci., 106, (1996), 301-328 and 421-449. 
[R2] A. Ramanathan: Stable principal bundles on a compact Riemann surface, Math. Ann. 213 (1975), 129-152.

[S] C. Simpson: Higgs bundles and Local systems, Pub. I.H.E.S. 75 (1992), 5-95. 